\title{
Genetic analysis of 2299delG and C759F mutations (USH2A) in patients with visual and/or auditory impairments
}

\author{
Elena Aller ${ }^{1,2}$, Carmen Nájera*, ${ }^{*}$, José $\mathrm{M}^{\mathrm{a}}$ Millán $^{2}$, Juan S Oltra ${ }^{2}$, Herminio Pérez-Garrigues ${ }^{3}$, \\ Concepción Vilela ${ }^{4}$, Amparo Navea ${ }^{5}$ and Magdalena Beneyto ${ }^{2}$
}

\author{
${ }^{1}$ Departamento de Genética, Facultad de C Biológicas, Universidad de Valencia, Valencia, Spain; ${ }^{2}$ Unidad de Genética y \\ Diagnóstico Prenatal, Hospital La Fe, Valencia, Spain; ${ }^{3}$ Servicio de Otorrinolaringología, Hospital La Fe, Valencia, \\ Spain; ${ }^{4}$ Unidad de Neurofisiología, Hospital La Fe, Valencia, Spain; ${ }^{5}$ Servicio de Oftalmología, Hospital La Fe, \\ Valencia, Spain
}

The most common mutation in the USH2A gene (Usherin), 2299delG, causes both typical Usher (USH) syndrome type II and atypical USH syndrome, two autosomal recessive disorders, characterised by moderate to severe sensorineural hearing loss and retinitis pigmentosa (RP). Furthermore, the C759F mutation in the USH2A gene has been described in $4.5 \%$ of patients with nonsyndromic recessive RP. We have investigated the presence of the 2299delG and/or the C759F mutations in 191 unrelated Spanish patients with different syndromic and nonsyndromic retinal diseases, or with nonsyndromic hearing impairment. The 2299delG mutation was observed in patients with clinical signs of USHII or of atypical USH syndrome, whereas the C759F mutation, regardless of being associated with the 2299delG mutation or not, was identified in cases with nonsyndromic RP, as well as in patients with RP associated with a variability of hearing impairment. The comparative analysis of both phenotypic and genotypic data supports the hypothesis that sensorineural hearing loss in patients with RP may depend on the nature and on the association of the USH2A allele variants present.

European Journal of Human Genetics (2004) 12, 407-410. doi:10.1038/sj.ejhg.5201138

Published online 18 February 2004

Keywords: 2299delG mutation; C759F mutation; USH2A gene; visual impairment; auditory impairment

\section{Introduction}

Usher syndrome (USH) is a group of autosomal recessive disorders characterised by the association of sensorineural hearing impairment and retinitis pigmentosa (RP). Three clinical types of USH have been described (MIM 276901, 276902, and 276903), where type II is the most common subtype. At least three USH2 loci (MIM 276901, 276905,

*Correspondence: Dr C Nájera, Departamento de Genética, Facultad C Biológicas, Universidad de Valencia, Dr Moliner 50, 46100 Burjasot, Valencia, Spain. Tel: + 34 963544506; Fax: + 34 963543029;

E-mail: Carmen.Najera@uv.es

Received 20 June 2003; revised 27 October 2003; accepted 30 October 2003 and 605472) have been mapped so far, although the USH2A locus, assigned to chromosome 1q41, is the locus most commonly responsible for USH syndrome type II. The USH2A gene (MIM 276901) was cloned in $1998 .^{1}$ This gene encodes Usherin, a protein that shows homology to both extracellular matrix proteins and receptors containing the laminin epidermal growth factor and fibronectin type III repeats. $^{1,2}$ The function of this protein is still unknown, but the expression of the USH2A gene has been detected by reverse transcriptase PCR (RT-PCR) performed on fetal human cochlea and eye, and also on adult human retina. ${ }^{1}$

A large number of different mutations have been identified, most of which were nonsense and frameshift. The most prevalent mutation found in the USH $2 A$ gene is 
the 2299delG. ${ }^{1-5}$ This mutation causes USH syndrome type II and also leads to atypical $\mathrm{USH}^{6}$ syndrome. Another frequent change in the USH2A gene, the C759F mutation, causes USHII syndrome ${ }^{4,7}$ and RP without hearing loss. ${ }^{8}$ Both mutations are located in exon 13, a region that represents only $6.4 \%$ of the coding frame.

Given the high allelic heterogeneity of this gene, the prevalence of these two mutations, and the phenotypic variation that these cause, the aim of this particular work was to investigate these changes in Spanish patients with typical USHII syndrome and atypical USH, as well as in patients with other nonsyndromic visual or auditory deficiencies such as RP and deafness. In order to investigate the phenotypic repercussion of these mutations, patients in whom only one mutation was detected were screened for the rest of the gene.

\section{Materials and methods Subjects}

We evaluated 191 unrelated patients from Spain suffering visual and/or hearing impairment. On the basis of clinical history, funduscopy examination and visual, neurophysiological, audiological and vestibular tests, these patients were classified into three groups. The first group was comprised of 76 probands, 59 of them being previously reported by us. $^{7}$ These patients were diagnosed with USHII syndrome, although a number of them referred to a subjective progressive hearing loss (atypical USH syndrome). The second group consisted of 80 probands diagnosed with recessive nonsyndromic RP. The last group was made up of 35 cases with nonsyndromic hearing impairment.

In all, 50 unrelated individuals of the Spanish population were screened as controls to evaluate the frequency of the mutations found in the patient sample.

\section{Mutation detection}

Genomic DNA was extracted from leucocytes from peripheral blood samples as described elsewhere. PCR was performed using primers under conditions described in previous studies. $^{2,7}$

An initial search for the 2299delG and C759F mutations was carried out in the newly ascertained patients. These mutations were detected by comparing the SSCP pattern of unknown alleles to the pattern of these known mutations.

A screening in the rest of the USH2A gene was performed with patients in whom, after the first search, the $2299 \mathrm{delG}$ or the C759F changes were only found in one of the two alleles. Mutation detection was performed by an SSCP analysis. Migration pattern variants were subsequently sequenced in an automated sequencer (ABI-PRISM 310, Applied Biosystems) in order to determine the nature of these sequence changes.

\section{Results and discussion}

The 2299delG and the C759F mutations were identified in 28 out of the 191 patients: four of them were homozygous for the 2299delG mutation, one patient was homozygous for the C759F change, and another one was a 2299delG/ C759F compound heterozygote. For this latter patient, segregation analysis showed the location of each mutation on different chromosomes. In the remaining 22 cases, only one mutated allele was detected after analysing exon 13: the 2299delG mutation was identified in 15 patients, whereas the $\mathrm{C759F}$ mutation was found in seven cases. The screening of the remaining USH2A exons led us to identify the second mutation in three of the 2299delG carriers and in two of the C759F carriers. Table 1 shows the distribution of these mutations among the groups established on the basis of their clinical features.

The two new mutations identified in this study (R303S and 3099-3100insTGAT) were not found in the controls. The frequency of the rest of mutations in our control population was described previously. ${ }^{7}$

Bernal et $a l^{9}$ found only one haplotype associated with the C759F mutation. In our sample, we detected at least two haplotypes associated with this mutation. This muta-

Table 1 Distribution of mutations among the groups established on the basis of the clinical characteristics

\begin{tabular}{|c|c|c|c|}
\hline Number of patients & Allele I & Allele II & Phenotype \\
\hline 1 & C759F & C759F & Nonsyndromic RP \\
\hline 3 & C759F & Unknown & Nonsyndromic RP \\
\hline 1 & C759F & 3099-3100insTGAT & Atypical USH \\
\hline $1^{\mathrm{a}}$ & C759F & Unknown & Atypical USH \\
\hline $1^{\mathrm{a}}$ & C759F & Unknown & USHIII \\
\hline $1^{\mathrm{a}}$ & C759F & $2431-2432$ delAA & USHII \\
\hline $4^{a}$ & 2299delG & 2299delG & USHII \\
\hline $11^{\mathrm{a}}, 1$ & 2299delG & Unknown & USHII \\
\hline 1 & 2299delG & 921-922insCAGC & USHII \\
\hline $1^{\mathrm{a}}$ & 2299delG & IVS12+5G > A & USHII \\
\hline 1 & 2299delG & R303S & USHII \\
\hline 1 & 2299delG & C759F & Atypical USH \\
\hline
\end{tabular}

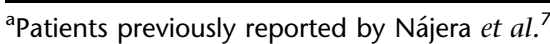


tion was only associated with the previously reported polymorphism, $\mathrm{H} 752 \mathrm{H}$, in eight out of ten $\mathrm{C} 759 \mathrm{~F}$ alleles. This could therefore suggest the existence of at least two different origins of C759F in the Spanish population.

The phenotype associated with the mutants detected in this particular study provides additional data among the reported interfamilial variation of both the severity and the onset of hearing loss in those individuals with mutations within the USH2A gene. Therefore, while the four homozygous patients with the 2299delG mutation suffered from typical USHII syndrome, clinical aspects of some of the remaining cases where both mutated alleles were identified offer certain peculiarities that are worth pointing out.

The homozygous C759F patient (64 years old) was diagnosed with nonsyndromic RP, and showed normal auditory evoked potentials. Rivolta et $a l^{8}$ reported two similar cases of homozygous $\mathrm{C} 759 \mathrm{~F}$ patients suggesting that $\mathrm{C} 759 \mathrm{~F}$ homozygosity causes recessive nonsyndromic RP without hearing loss. However, Bernal et al ${ }^{9}$ reported two asymptomatic sibs homozygotes for this missense mutation, which may indicate that homozygosity for C759F does not always cause disease.

Four of the six compound heterozygote patients reported in this study (C759F/2431-2432delAA, 2299delG/921922insCAGC, 2299delG/IVS12 + 5G > A, 2299delG/R303S) suffered from typical USHII, whereas the remaining two cases (2299delG/C759F and C759F/3099-3100insTGAT) corresponded to the atypical USH syndrome. Neither familial nor personal history was suggestive of any cause of the hearing loss in any of these two atypical cases. Nor did they have histories suggestive of equilibrium pathology. Otoscopic exploration and impedanciometry were normal in both cases. The electronystagmography was also normal with regular rotatory and caloric vestibular exploration tests. A radiological study of the VIII cranial nerve canal was also normal.

The 2299delG/C759F patient underwent an audiometry, which showed a progressive sensorineural hearing loss, somewhat more noticeable in the left ear (Figure 1). Speech audiometry (a dichotic speech test) showed a discrimination of $95 \%$ in the right ear at $60 \mathrm{db}$, and $85 \%$ in the left ear at $90 \mathrm{db}$.

The patient with the C759F/3099-3100insTGAT mutation was initially diagnosed with nonsyndromic RP. However, when he reached the age of 52, he showed slightly abnormal auditory evoked potentials. At 55 years old, his auditory evoked potentials were abolished. This would indicate a rapid progression of his hearing impairment. Unfortunately, this patient did not wish to collaborate and did not undergo an audiometric test.

Rivolta et $a l^{8}$ identified two recessive nonsyndromic RP patients who were compound heterozygotes with $\mathrm{C} 759 \mathrm{~F}$ and frameshift mutations (2299delG and 43384339delTC); Bernal et $a l^{9}$ found additional compound heterozygotes with $\mathrm{C} 759 \mathrm{~F}$ and nonsense, splicing or missense mutations associated with phenotypic characteristics of nonsyndromic RP, reinforcing the hypothesis that mutations in the USH2A gene can result in ARRP without a loss of hearing. Nevertheless, three cases with C759F associated with three different frameshift mutations reported in this study suffer from RP in association with hearing impairment: the C759F/2431-2432delAA patient (typical USHII syndrome) plus those with C759F/2299delG and C759F/3099-3100insTGAT (both atypical USH syn- a
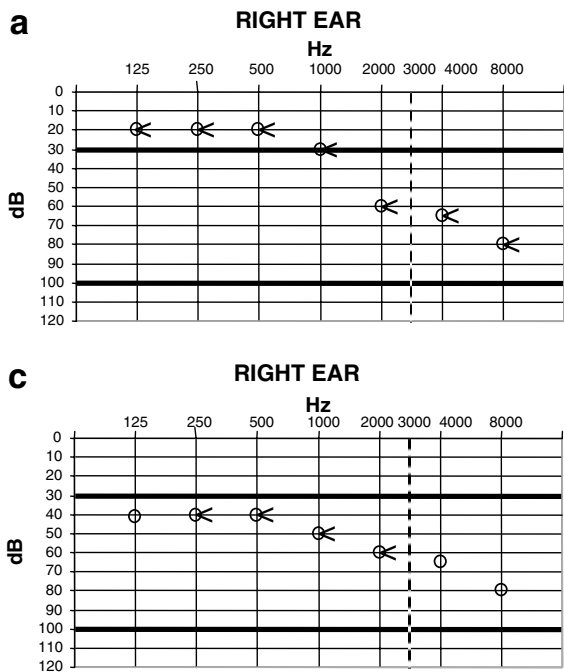

b

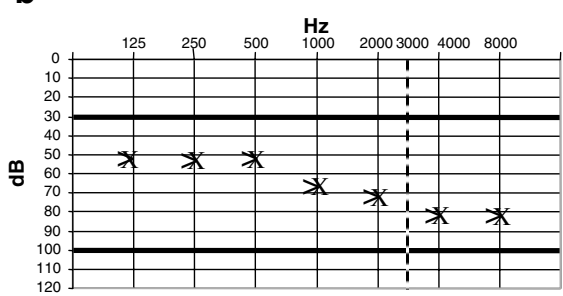

d

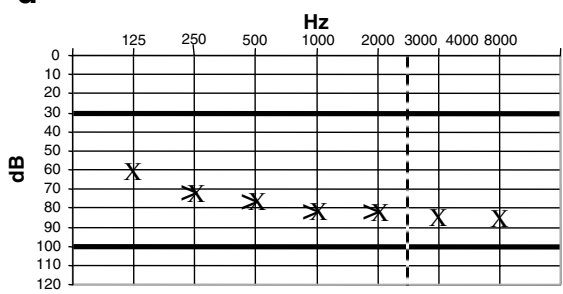

Figure 1 Pure-tone audiograms of patient carrying 2299delG/C759F mutations. O represents air conduction threshold measurements; right ear. $X$ represents air conduction threshold measurements; left ear. $<$ represents bone conduction threshold measurements; right ear. > represents bone conduction threshold measurements; left ear. A and B show pure-tone audiograms at the age of 50 years old. $C$ and D show pure-tone audiograms at the age of 53 years old. 
drome). This suggests that 2299delG and C759F mutations could be responsible for a wide variability in sensorineural hearing loss. This phenotypic variability may be explained by the combination of either these two mutations or any of them with other mutations of the USH2A gene. For this reason we recommend that all those cases with nonsyndromic RP and a mutation within the USH $2 A$ gene should undergo a highly sensitive ENT examination to verify the auditory function.

In probands with nonsyndromic hearing impairment, neither 2299delG nor C759F has been detected. This is of no surprise since none of the loci and genes related to nonsyndromic deafness are located in the 1q41 region, where the USH2A gene maps.

A wide range of phenotypes in patients with Usherin mutations have been described; there have even been reports on cases carrying the same mutations associated with different clinical signs: homozygosity for C759F has been described in nonsyndromic RP cases (Rivolta et al, ${ }^{8}$ present report) and in asymptomatic cases; ${ }^{9}$ homozygosity for 2299delG has been described in two monozygotic twins, one of them suffering the typical USHII syndrome and the other one suffering the atypical USH syndrome; ${ }^{6}$ compound heterozygotes C759F/2299delG have been described as nonsyndromic $\mathrm{RP}^{8}$ and as atypical USH syndrome (present report). These findings may indicate that the nature of the mutations along with other genetic or environmental factors are involved in the phenotype of the USH2A gene mutations. This fact confirms the need for further molecular and biochemical studies to be carried out, along with phenotype-genotype correlation studies, to elucidate the function of this protein in the retina and in the cochlea.

\section{Acknowledgements}

We thank the participating patients and their family members, and also the FAARPEE for their help and cooperation. This work was supported by funds from Fondo de Investigaciones Sanitarias (FIS 98/ 0338, 01/0081-02), ONCE, Fundación ONCE, and Fundaluce.

\section{References}

1 Eudy JD, Weston MD, Yao S et al: Mutation of a gene encoding a protein with extracellular matrix motifs in Usher syndrome type IIa. Science 1998; 17: 1753-1757.

2 Weston MD, Eudy JD, Fujita S et al: Genomic structure and identification of novel mutations in Usherin, the gene responsible for Usher syndrome type IIa. Am J Hum Genet 2000; 66: $1199-1210$

3 Beneyto M, Cuevas JM, Millán JM et al: Prevalence of 2314delG mutation in Spanish patients with Usher syndrome type II (USH2). Ophthalmic Genet 2000; 21: 123-128.

4 Dreyer B, Tranebjaerg I, Rosemberg T, Weston M, Kimberling W, Nilsen O: Identification of novel USH2A mutations: implications for the structure of USH2A protein. Eur J Hum Genet 2000; 8: 500-506.

5 Leroy BP, Aragon-Martin JA, Weston MD et al: Spectrum of mutations in USH2A in British patients with Usher syndrome type II. Exp Eye Res 2001; 72: 503-509.

6 Liu XZ, Hope C, Liang CY et al: A mutation in the Usher syndrome type IIA gene: high prevalence and phenotypic variation. Am J Hum Genet 1999; 64: 1221-1225.

7 Nájera C, Beneyto M, Blanca J et al: Mutations in Myosin VIIA (MYO7A) and Usherin (USH2A) in Spanish patients with Usher syndrome types I and II, respectively. Hum Mutat 2002; 20: $76-77$.

8 Rivolta C, Sweklo EA, Berson EL, Dryja TP: Missense mutation in USH2A gene: association with recessive retinitis pigmentosa without hearing loss. Am J Hum Genet 2000; 66: 1975-1978.

9 Bernal S, Ayuso C, Antiñolo G, Gimenez A, Borrego S, Trujillo MJ et al: Mutations in USH2A in Spanish patients with autosomal recessive retinitis pigmentosa: high prevalence and phenotypic variation. J Med Genet 2003; 40: e8. 